\title{
Shakedown analysis of 3D frames under multiple load combinations using mixed fiber beam elements
}

\author{
D. Magisano*, L. Leonetti* and G. Garcea* \\ * Dipartimento di Ingegneria Informatica, Modellistica, Elettronica e Sistemistica \\ Università della Calabria, 87030 Rende (Cosenza), Italy
}

\begin{abstract}
If the stress intensity at a certain point of a structure made of ductile materials reaches the yield point, the structure does not necessarily fail or deform excessively. Instead, stress redistribution could take place and some further load increments could be supported. Thus, the actual loadcarrying capacity of a structure is generally higher than the elastic limit and this overstrength can be exploited for a cost-effective design. However, during their operational life, structures are subjected to variable loads, whose law of variability in time is often unknown. Instead, it is usually possible to have a good estimate of the variability range in terms of maximum and minimum load amplitude. Within this context, shakedown analysis is able to provide a reliable safety factor against plastic collapse, loss in functionality due to excessive deformations (ratcheting) or collapse due to low cycle fatigue (plastic shakedown). This work presents an efficient fiber analysis for evaluating the shakedown safety factor of 3D frames under multiple load combinations. Mixed beam elements are employed for an accurate discretization of the structures [1]. A continuation method, similar to an incremental elasto-plastic analysis, is used at structural level. It evaluates a fictitious equilibrium path made of a sequence of safe states with a converging non-decreasing load factor [2]. Each point of the path is obtained by finding kinematic variables corresponding to self-equilibrated stresses satisfying Melan's condition for the current load factor to be safe. The stress admissible domain is defined at fiber level as a function of the load factor using the maximum and minimum effect due to all loads [3]. An iterative state determination provides finite element stresses corresponding to assigned kinematic variables and load factor [3]. The overall analysis differs from previous proposals for two novelty points. Firstly, a direct application of the Newton method is possible, without any need for constrained optimization solvers. Moreover, dimension and complexity of the load domain does not affect the computational cost of the nonlinear analysis. Numerical tests highlight an accurate estimate of the safety factor using a small number of fibers and an efficient solution also for large buildings.
\end{abstract}

\section{REFERENCES}

[1] Leonardo Leonetti, Raffaele Casciaro, Giovanni Garcea, Effective treatment of complex statical and dynamical load combinations within shakedown analysis of 3D frames. Computers Es Structures (2015) 158:124-139.

[2] Raffaele Casciaro, Giovanni Garcea, An iterative method for shakedown analysis. Computer Methods in Applied Mechanics and Engineering (2002) 191:5761-5792.

[3] Magisano, D, Garcea, G. Fiber-based shakedown analysis of three-dimensional frames under multiple load combinations: Mixed finite elements and incremental-iterative solution. Int $J$ Numer Methods Eng. (2020) 121:3743-3767. 